\title{
Endoscopic Magnetic Resonance Imaging at Variable Coil Orientations
}

\begin{abstract}
Amongst the latest developments in gastrointestinal magnetic resonance (MR) imaging has been the design of a non-ferromagnetic endoscope that has all the features of a standard forwardviewing endoscope (1). Incorporated into the tip of the MR endoscope is a rectangular wire loop $(10 \times 30 \mathrm{~mm})$ that acts as the MR receiver coil. The images obtained show a high spatial resolution, and are able to demonstrate three to five distinct histologic layers in the gastrointestinal wall (2). Preliminary studies in patients with esophageal, gastric, and rectosigmoidal disease have shown promising results with regard to the local staging of gastrointestinal tumors $(3,4)$. However, the investigators observed that scanning in the gastric antrum or the sigmoid colon, where the MR endoscope coil is not aligned with the longitudinal axis of the magnetic field of the MR scanner, yielded insufficient image quality. We systematically assessed the sensitivity profile of the coil at different orientations.
\end{abstract}

Imaging was performed on a 1.5 -Tesla scanner using a fast-spoiled gradient sequence. The endoscope tip was placed in a plastic container filled with copper sulfate solution. The coil was positioned at first in a vertical plane, and then in a horizontal plane (Figure 1). At both orientations, imaging was performed with the coil at $0^{\circ}, 30^{\circ}, 45^{\circ}, 60^{\circ}$, and $90^{\circ}$ angles compared to the longitudinal axis of the MR scanner.

When the coil was aligned with the magnetic field, the crosssectional sensitivity distribution had a circular shape, which was unchanged at $30^{\circ}$ (Figure 2). At a coil angulation of $45^{\circ}$ or more, however, the sensitivity profile became gradually distorted into a four-leafed clover shape, leaving areas without signal between the lobes. Imaging with the loop coil oriented in a vertical or in a horizontal plane both showed similar four-leafed clover shape profiles, but rotated by $45^{\circ}$ in relation to one another. This suggests that combining the signals from two such coils, set at a $90^{\circ}$ angle to each other and operated simultaneously (quadrature design), would provide a solution to this problem. A computer simulation of this configuration shows that the two profiles perfectly complement one another, and an almost uniform sensitivity pattern can be achieved (Figure 3 )

The clinical observation of inadequate image quality when the MR endoscope is placed in the gastric antrum or the sigmoid colon $(3,4)$ can be explained by distortion of the sensitivity profile, with areas of signal void at oblique orientations of the MR coil. The use of a quadrature coil design would allow proper imaging at any position of the MR endoscope. This would expand the variety of indications for this new instrument, with particular implications for pancreatic imaging (5).

D. Kulling', D. E. Bohning ${ }^{2}$, C. L. Kay ${ }^{2}$, D. R. Feldman²,

R. H. Hawes'

' Dept. of Gastroenterology

${ }^{2}$ Dept. of Radiology, Digestive Disease Center, Medical

University of South Carolina, Charleston, South Carolina, USA

\section{References}

1. Feldman DR, Kulling D, Hawes RH, Kay CL, Muckenfuss VR, Cotton PB, et al. MR endoscopy: preliminary experience in human trials. Radiology 1997; 202: 868-70.

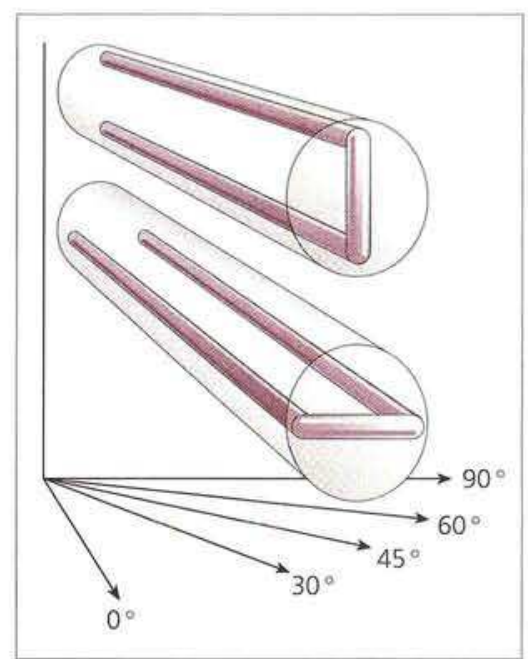

Figure 1: Imaging was carried out at five different angles.

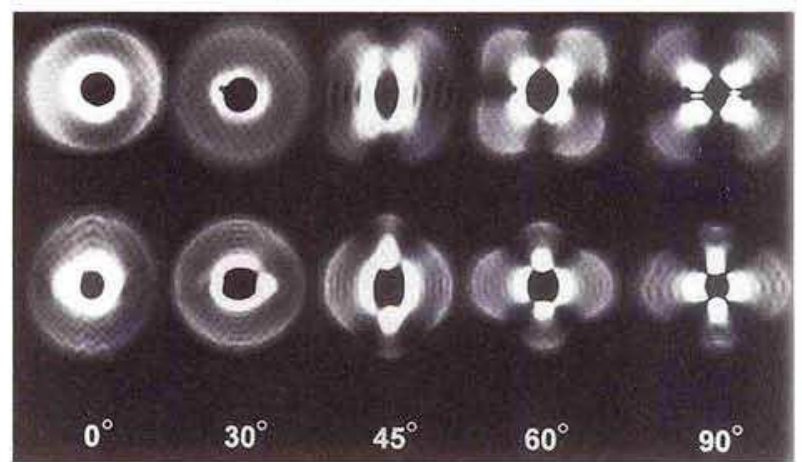

Figure 2: The sensitivity profiles with the coil in a vertical plane (upper row) and a horizontal plane (lower row)

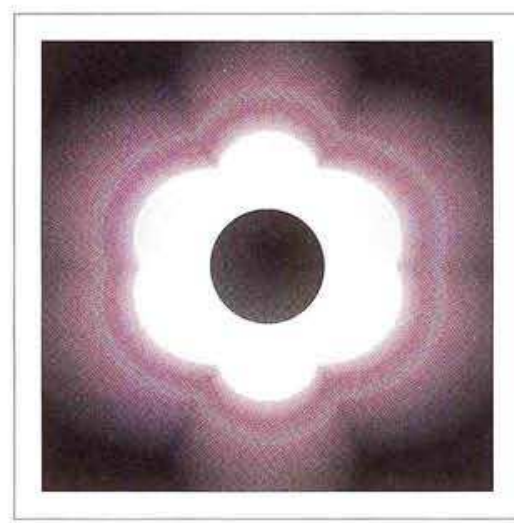

Figure 3: Simulated field distribution for the quadrature coil at a $90^{\circ}$ angulation

2. Kulling D, Bohning DE, Kay C, Spicer K, Hawes R. Identification and histologic correlation of gastrointestinal wall layers imaged with an MR endoscope. Am J Gastroenterol 1996; 91 : 1990. 
3. Inui K, Nakazawa S, Yoshino J, Yamao K, Yamachika H, Wakabayashi T, et al. Endoscopic MRI: preliminary results of a new technique for visualization and staging of gastrointestinal tumors. Endoscopy 1995; 27: 480-5.

4. Murano A, Sasaki F, Kido C, Nakamura T, Kobayashi S, Kato T, et al. Endoscopic MRI using 3 D-spoiled GRASS (SPGR) sequence for staging of rectal carcinoma. J Comput Assist Tomogr 1995; 19: 586-91.
5. Moriya H, Yoshitome E, Nagasawa K, Inui K, Nakazawa S. Development of the MR endoscope and its clinical applications. ISMRM Proc 1996: 1438.

Corresponding Author

D. Kulling, M.D., Digestive Disease Center

Medical University of South Carolina

171 Ashley Avenue, Charleston, SC 29425, USA

Fax: $+1-803-792-4184$ 